\title{
TRATAMIENTO DE LAS INFECCIONES MALARICAS PRODUCIDAS POR EL PLASMODIUM FALCIPARUM Y EL PLASMODIUM VIVAX
}

\author{
CARLOS A. ESPINAL TEJADA *
}

\section{INT R ODUCCION}

El tratamiento adecuado de la malaria ha presentado múltiples dificultades por razones que involucran la certeza del diagnóstico, la resistencia del P. falciparum a la cloroquina y a otros antimaláricos y el poco conocimiento del personal médico y paramédico, aún en áreas de alta transmisión, de uno de los problemas importantes de Salud Pública en Colombia.

Los primeros indicios de resistencia del $P$. falciparum a la cloroquina fueron informados por Moore y Young en 1961 $(1,2)$, en infecciones provenientes del Valle del Magdalena. Estudios posteriores in-vivo e in-vitro, $(3,4)$, han confirmado la presencia de infecciones en diversos grados de resistencia en diferentes regiones del pais; sin embargo, la distribución geográfica de las áreas cloroquina-resistentes y el grado de $\mathbf{P}$. falciparum a los antimaláricos deben ser determinados a través de estudios epidemiológicos representativos, con el fin de obtener una información básica para la elección del tratamiento y el seguimiento de la infección.

La resistencia del $P$. falciparum a la cloroquina es clasificada en grados siguiendo la respuesła de los parásitos asexuados a una dósis determinada de la droga. Las infecciones son sensibles (S), si no se encuentran parásitos circulantes en un período de 7 días post-tratamiento; si los anillos desaparecen de la sangre periférica pero retornan en un lapso de 28 días, la infección se clasifica como resistente al nivel RI; la marcada reducción de

Médico Unidad Inmunología de Malaria. Grupo de Microbiología e Inmunología. Instituto Nacional de Salud la parasitemia sin desaparición completa de los anillos, representa el nivel RII; en el nivel RIII no hay reducción o inclusive la densidad parasitaria aumenta en presencia de la droga, (5).

La presente publicación revisa algunos de los conceptos básicos en el diagnóstico y tratamiento de la malaria, con énfasis especial en las infecciones por el P. falciparum resistente a la cloroquina.

\section{DIAGNOSTICO MICR OSCOPICO}

El análisis microscópico de sangre periférica es el método de elección en el diagnóstico de la malaria. Tanto el extendido como la gota gruesa son procedimientos que deben ser incluidos en las muestras iniciales de un paciente con un cuadro clínico compatible con la enfermedad. La gota gruesa concentra la densidad parasitaria y permite el hallazgo rápido aún en infecciones con bajo número de parásitos/m.c.l.sangre. El extendido de sangre periférica es utilizado en la identificación de especies cuando no es posible hacerlo en la gota gruesa y en la cuantificación de la parasitemia en infecciones con recuentos altos de parásitos.

El número de parásitos (formas asexuadas) /m.c.lsangre es un factor determinante en la selección del tratamiento y en su evolución ya que permite conocer la actividad real de los antimaláricos en las primeras 48 horas, además de indicar el grado de susceptibilidad de la infección a la terapia establecida. El recuento de parásitos/m.c.l.sangre puede obtenerse en gota gruesa y extendido, al determinar el número de formas asexuadas por 300 glóbulos blancos o en 10.000 glóbulos rojos respectivamente. 
TR ATAMIENTO DE L. A INFE CCION AGUDA POR P. F AL CIPAR UM:

El cuadro clínico de la infección por $\mathbf{P}$ falciparum es muy variado y depende del nivel de inmunidad del huésped, la susceptibilidad del parásito a los antimaláricos, la duración e intensidad de la parasitemia y la presencia de complicaciones (cerebrales, renales, hematológicas, etc.). La infección, al progresar rápidamente debido a la multiplicación masiva de las formas asexuadas, es potencialmente letal y los casos complicados deben ser considerados como emergencias médicas, La selección del tratamiento debe hacerse de acuerdo con el número de parásitos/m.r.l.sangre, el área geográfica de origen de la infección y el estado clínico e inmunitario del paciente.

A. Infecciones Sensibles a las 4. Aminoquinolinas: Las áreas maláricas con sensibilidad del $\mathbf{P}$. falciparum a la cloroquina, se han visto reducidas debido a la rápida dispersión de cepas resistentes en las nuevas áreas de colonización. A pesar de la amplia distribución del fenómeno, aún se encuentran infecciones sensibles en algunas regiones de las Costas Atlántica y Pacífica y un bajo porcentaje en el interior del país, que puedan ser tratadas con el esquema tradicional presentado en el Cuadro No. 1. Como puede observarse, la determinación del número de parásitos/m.c.l.sangre en la muestra inicial del paciente, representa el límite entre las infecciones agudas complicadas y las de fácil manejo clínico, pudiéndose presentar el cuadro severo de la enfermedad en algunos casos con número de parásitos inferior a los 100.000 /m.r.l. sangre.

Los pacientes con recuentos superiores a 50.000 e inferiores a $100.000 /$ m.c.l. sangre desarrollan frecuentemente episodios de vómito y/o diarrea, necesitando la administración parenteral de la cloroquina. Una dósis de $200 \mathrm{mg}$. de cloroquina (base) cada 6 horas, por vía intramuscular, con un límite máximo de $900 \mathrm{mg}$. en 24 horas, será suficiente para el control adecuado de la infección, con la posibilidad de continuar el tratamiento por vía oral tan pronto como las condiciones del paciente lo permitan. La dósis pediátrica de cloroquina debe ser calculada con base en el peso corporal y no debe ser administrada por via parenteral en niños menores de 5 años, a menos que sea absolutamente indispensable. Cuando su aplicación intramuscular se haga necesaria, la dosis no debe exeder los 5 $\mathrm{mg} / \mathrm{kg}$ de peso, en un período de 24 horas, ya que por su toxicidad puede inducir hipotensiones severas con muerte súbita (6).

El control de los gametocitos es indispensable con el fin de interrumpir la cadena de transmisión de la enfermedad. Una dósis

\section{CUADRO I}

PLASMODIUM FALCIPARUM

INFECCIONES SENSIBLES A LAS 4 - AMINOQUINOLINAS

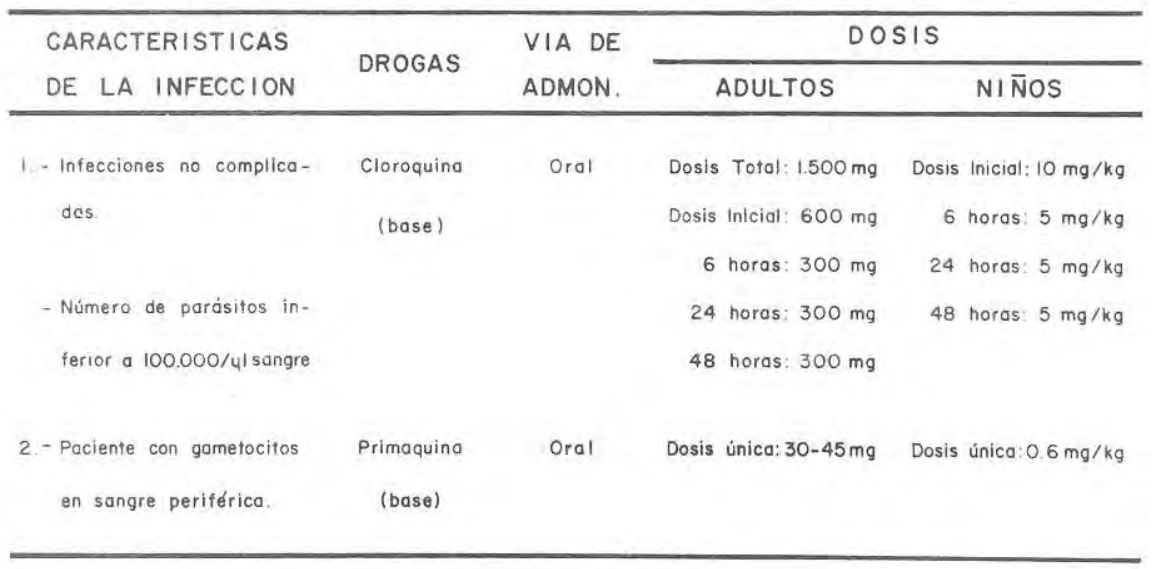


única de $30-45 \mathrm{mg}$. de primaquina (base), esteriliza completamente las formas sexuadas, aunque niveles bajos de gametocitemia pueden persistir en la sangre periférica por una o dos semanas.

B. Infecciones R esistentes a las 4 - Ami. noquinolinas: El bajo grado de susceptibilidad del P. falciparum a la cloroquina ha sido demostrado con pruebas in-vitro en algunas áreas del país (Fig. 1). Las cepas sensibles a las 4 - Aminoquinolinas inhiben su maduración de esquizontes en 5 picomoles de la droga, mientras que las resistentes, como las detectadas en Vista Hermosa, Departamento del Meta, tienen su máxima inhibición en 32-64 picomoles de cloroquina, indicando un nivel RII-RIII de resistencia (datos no publicados). El tratamiento de estas infecciones esquematizado en el Cuadro No. 2, presenta diferentes alternativas de acuerdo con las características del cuadro clínico del paciente, el grado de resistencia del parásito a la cloroquina y la inmunidad conferida por previas infecciones en personas residentes en áreas maláricas.

Como puede observarse, la administración prolongada de sulfato de quinina por un periodo superior a los 3 días no está incluida en los esquemas propuestos, debido a su alta intolerancia y efectos colaterales; uno de los principales es el denominado cinconismo caracterizado por náuseas, vómito y tinnitus, que puede presentarse al recibir la droga łanto por vía venosa como por vía oral. El esquema tradicional de $2 \mathrm{gm} /$ día por 14 dias, únicamente se recomienda en los casos de recrudescendencia a los múltiples tratamientos descritos con anterioridad.

La combinación en dósis única de fanasil y pirimetamina bloquea la síntesis del ácido folínico. Las sulfonamidas interfieren con la utilización del PABA requerido para la síntesis del dihidrofolato, y la pirimentamina se combina irreversiblemente con la enzima dihidrofolato reductosa, necesaria para la formación del tetrahidrofolato (ácido folínico). Este sinergismo es muy efectivo en la eliminación del parásito, pero debe asociarse siempre a un esquizonticida sanguíneo de acción rápida del tipo de la quinina o amodiaquina, con el fin de controlar efectivamente la infección. Solamente en pacientes semi-inmunes con número bajo de parásitos 向.c.lsangre, es recomendable la administración de sulfa y pirimentamina como única medicación. En el caso de infecciones provenientes de áreas con predominio de RI y RII o en ausencia del sulfato de quinina, el tratamiento puede iniciarse con amodiaquina, asociada a la sulfa-pirimetamina.

Estudios preliminares de Schmidt y colaboradores, (7), en primates del género Aotus infectados con P. falciparum, demostraron la susceptibilidad a la amodiaquina en dos cepas resistentes a la cloroquina. Posteriormente Rieckmann, en 1971, confirmó esłos hallazgos en personas infectadas con cepas resistentes RIII a la cloroquina. Con la administración de $1.500 \mathrm{mg}$. de amodiaquina (base), la parasitemia desapareció de la sangre periférica, pero la recrudescencia fue observada 13 días después del inicio del tratamiento (nivel de RI a la amodiaquina), (8). Las 4 - Aminoquinolinas suprimirán la parasitemia circulante y los antifolínicos las formas resistentes que harán la recrudescencia posteriormente.

La resistencia múltiple del P . falciparum a diversas drogas en un fenómeno que se ha venido presentando con mayor frecuencia. Son varios los casos documentados y tratados en la Unidad de Inmunología de Malaria que han demostrado resistencia a la cloraquina y a la combinación de sulfas y pirimetamina, asociada o no a la quinina. Estas infecciones solamente han sido erradicadas con la administración de la quinina y las tetraciclinas a las dosis recomendadas, ofreciendo una efectividad completa en los casos tratados. La medicación con pirimetamina debe ajustarse a la dósis por kilogramo peso, ya que las sobredosis pueden traer serias complicaciones como la leucopenia, disminución del hematocrito y la hemoglobina y cambios megaloblásticos a nivel de la médula ósea. No existe evidencia clínica o experimental en la inducción de estos cambios con las dosis recomendadas para el tratamiento y la profiliaxis de la malaria, (9). Sin embargo, en nuestro medio, los factores nutricionales con deficiencia de ácido fólico, pueden aumentar 
la susceptibilidad de los individuos a los efectos tóxicos de la droga. La amplia utilización del Methipox (500 mg. de sulfadimetoxina y $25 \mathrm{mg}$. de pirimetamina) en las áreas maláricas, hace de la automedicación una de las causas más comunes en la aparición de estos efectos adversos de la pirimetamina y es un fenómeno que el médico debe tener presente, antes de establecer el tratamiento indicado.

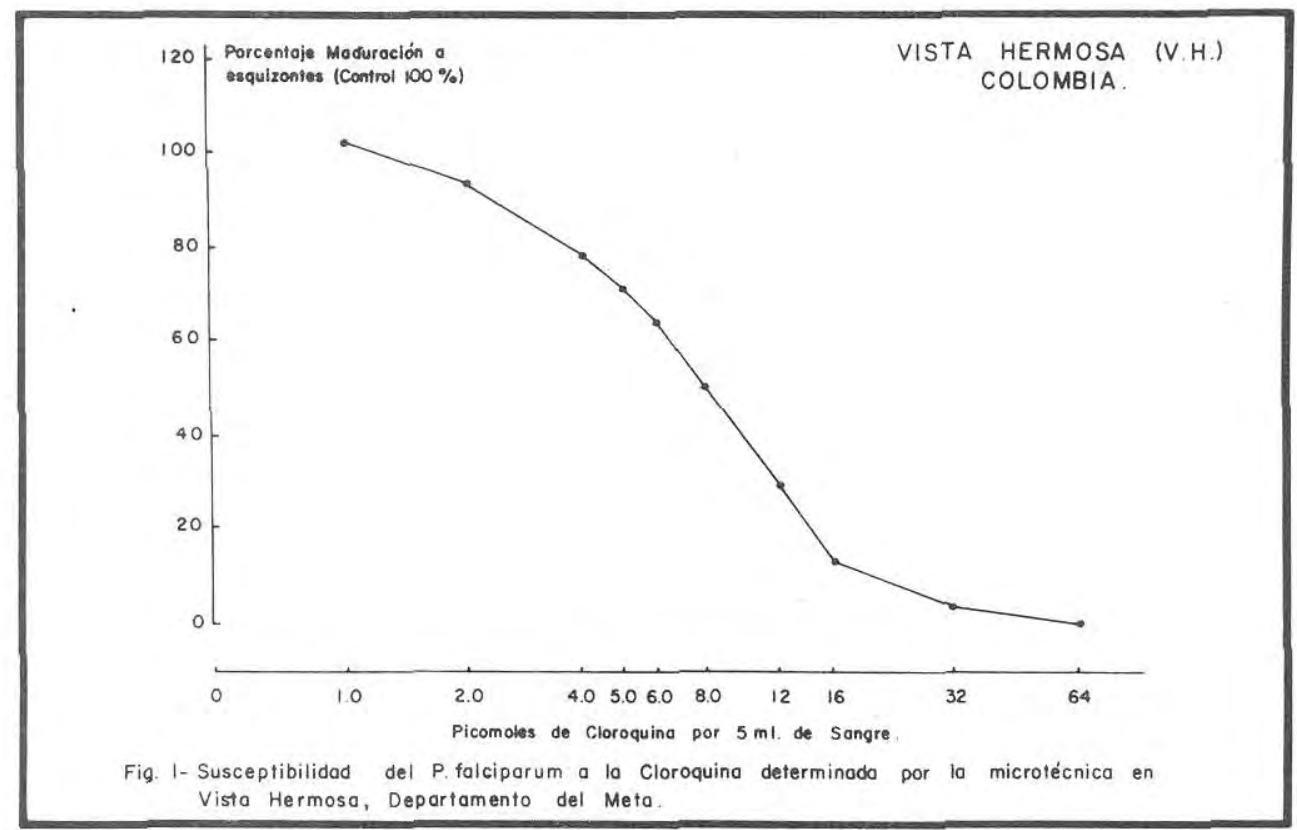

PLASMODIUM FALCIPARUM INFECCIONES RESISTENTES A LASS 4 - AMINOQUINOLINAS

\begin{tabular}{|c|c|c|c|c|}
\hline \multirow{2}{*}{$\begin{array}{l}\text { CARACTERISTICAS } \\
\text { DE LA INFECCION }\end{array}$} & \multirow{2}{*}{ DROGAS } & \multirow{2}{*}{$\begin{array}{l}\text { VIA DE } \\
\text { ADMON. }\end{array}$} & \multicolumn{2}{|c|}{ DOSIS } \\
\hline & & & AOULTOS & NIÑOS \\
\hline 1. Infecciones no complicadas. & Sulfato de Quinina. & Oral & $\begin{array}{l}10 \mathrm{mg} / \mathrm{kg} / \text { cada } 8 \text { horas } \\
\text { por } 3 \text { dias. }\end{array}$ & $\begin{array}{l}10 \mathrm{mg} / \mathrm{kg} / \text { cado } 8 \text { horos } \\
\text { por } 3 \text { dias. }\end{array}$ \\
\hline $\begin{array}{l}\text { - Número de parásitos inferior a } \\
100000 / 41 \text { sangre } \\
\text { - No vómito y/0 diarrea. } \\
\text { - Recrudescencias de infecciones tra } \\
\text { tadas como sensibles. }\end{array}$ & $\begin{array}{l}\text { Sulfadoxina (Fonasil) } \\
\text { Pirimetamina }\end{array}$ & $\begin{array}{l}\text { Oral } \\
\text { Oral }\end{array}$ & $\begin{array}{l}\text { Dosis única: } 1.500 \mathrm{mg} \\
\text { Dosis única: } 75 \mathrm{mg}\end{array}$ & $\begin{array}{l}\text { Dosis única: } 25 \mathrm{mg} / \mathrm{kg} \\
\text { Dosis única: } 1 \mathrm{mg} / \mathrm{kg}\end{array}$ \\
\hline $\begin{array}{l}2 \text { Pacientes con antecedentes de in - } \\
\text { fecciones por P. folciparum. }\end{array}$ & $\begin{array}{l}\text { a) Amodiaquina (Dose) } \\
\text { Sulfodoxina (Fanasil) } \\
\text { Pirimetamina }\end{array}$ & $\begin{array}{l}\text { Oral } \\
\text { Oral } \\
\text { Oral }\end{array}$ & $\begin{array}{l}\text { Dosis total: } 1.500 \mathrm{mg} * \\
\text { Dosis única: } 1.500 \mathrm{mg} \text {. } \\
\text { Dosis única: } 75 \mathrm{mg}\end{array}$ & $\begin{array}{l}\text { Dosis total: } 25 \mathrm{mg} / \mathrm{kg} \text { * } \\
\text { Dosis única: } 25 \mathrm{mg} / \mathrm{kg} \\
\text { Dosis única: } 1 \mathrm{mg} / \mathrm{kg}\end{array}$ \\
\hline $\begin{array}{l}\text { - Número de parásitos inferior a } \\
20000 / 41 \text { sangre. }\end{array}$ & $\begin{array}{l}\text { b) Sulfadoxina (Fanasil) } \\
\text { Pirimetamina }\end{array}$ & $\begin{array}{l}\text { Oral } \\
\text { Oral }\end{array}$ & $\begin{array}{l}\text { Dosis única: } 1.500 \mathrm{mg} \\
\text { Dosis única: } 75 \mathrm{mg} \text {. }\end{array}$ & $\begin{array}{l}\text { Dosis única: } 25 \mathrm{mg} / \mathrm{kg} \\
\text { Dosis única: } 1 \mathrm{mg} / \mathrm{kg}\end{array}$ \\
\hline $\begin{array}{l}\text { 3. Pacientes con recrudescencia del } \\
\text { P. falciparum a los esquemas ex- } \\
\text { puestos. }\end{array}$ & $\begin{array}{l}\text { a) Sulfato de Quinina } \\
\text { Tetraciclinas. } \\
\text { b) Amodiaquina (base) } \\
\text { Tetraciclinas. }\end{array}$ & $\begin{array}{l}\text { Oral } \\
\text { Oral } \\
\text { Oral } \\
\text { Oral }\end{array}$ & $\begin{array}{l}10 \mathrm{mg} / \mathrm{kg} / \text { cada } 8 \text { horas, } \\
\text { por } 3 \mathrm{dias} \text {. } \\
250 \mathrm{mg} / \text { cada } 6 \text { horas / } \\
14 \mathrm{dios} \text {. } \\
\text { Dosis total: } 1.500 \mathrm{mg} * \\
250 \mathrm{mg} / \mathrm{cada} 6 \text { horas } \\
14 \text { dias. }\end{array}$ & \\
\hline
\end{tabular}

* La dosificación y el esquema de administración de la amodiaquina es similar a la cloroquina. 
C. Infecciones Agudas y Complicadas inducidas por el P. Falciparum : La infección aguda por $\mathbf{P}$. falciparum puede presentar diversos tipos de complicaciones que incluyen el cuadro cerebral, la insuficiencia renal aguda (IRA), la hepatitis malárica, muy severa en algunos casos, el edema pulmonary una serie de manifestaciones hematológicas diversas que pueden colocar al paciente en un alto riesgo de muerte. Las infecciones con número de parásitos superior a $100.000 / m$.r. sangre aún sin presentar ninguna de las características anteriores, deberán considerarse como críticas, pues en pocas horas el paciente puede desarrollar algunas de las formas severas de la enfermedad, debido a las altas parasitemias alcanzadas durante la rápida multiplicación de las formas asexuadas.

El Cuadro No. 3 resume el tratamiento de la infección aguda de acuerdo a las características clínicas del paciente, cubriendo asi las infecciones tanto sensibles como resistentes a la cloroquina.

Al iniciar la terapia antimalárica en estos tipos de complicaciones debe tenerse un control muy estricto del número de parásitos /m.s.l. sangre cada 12 horas, con el fin de determinar la efectividad del tratamiento y prevenir problemas adicionales, al incrementarse la parasitemia por una pobre respuesta a las drogas. La quinina, tiene una vida media de 10 horas en personas normales y la dosis puede continuarse por varios días con un máximo de 12 dósis en infusión intravenosa lenta. Los pacientes comatosos pueden recibir como terapia adicional 3-10 mg. de dexametasona cada 8 horas y 1 unidad de dextrán 75 cada 12 horas, reduciendo el edema cerebra! y mejorando la alteración vascular.

La insuficiencia renal aguda (IRA) es una complicación severa de la infección malárica y es recomendable medir la eliminación urinaria en pacientes hospitalizados con número de parásitos superior a $150.000 /$ m.c.l. sangre, ya que el acumulo de las formas maduras (trofozoitos y asegmentos) en la microcirculación, la hipotensión y el depósito de complejos inmunes a nivel del glomérulo, pueden desencadenar el sindrome y agravar el estado general del paciente. La vida media de la quinina es más prolongada en estos pacientes y concentraciones tóxicas de la droga pueden ser alcanzadas con las dosis convencionales. Asimismo, en la disfunción hepática por $\mathbf{P}$. falciparum, la rata metabólica de la quinina parece ser inversamente proporcional a la severidad de la infección; por lo tanto, la dósis óptima debe reducirse a $10 \mathrm{mg} / \mathrm{kg} / \mathrm{dia}$, con un control adecuado de los líquidos parenterales en ambas situaciones.

Debido a la amplia distribución de las cepas de $\mathbf{P}$. falciparum resistentes a la cloroquina y probablemente a otros antimaláricos, se recomienda complementar con la combinación de las sulfas y pirimetamina, una vez el paciente tolere la vía oral. No existen bases para un posible efecto potenciador de la quinina asociada a la sulfa-pirimetamina; además, debido a la acumulación de los parásitos maduros en la microcirculación de las paredes del tracto gastrointestinal, el paciente tiene dificultades en la absorción de las drogas, reduciendo su efectividad terapéutica. Por estos motivos no es recomendable la administración de drogas antimaláricas diferentes a la quinina intravenosa, hasta tanto el paciente no tenga una adecuada tolerancia por la vía oral.

\section{TR AT AMIE NTO DE LAS INFE CCIONES POR P. VIVAX}

El tratamiento del $\mathbf{P}$. vivax requiere drogas que actúen a nivel del ciclo eritrocítico, como las 4-Aminoquinolinas, y medicamentos del grupo de las 8-Aminoquinolinas, como el difosfato de primaquina, que actúan a nivel del ciclo exoeritrocítico ejerciendo su acción sobre los esquizontes hepáticos de evolución lenta. (Cuadro No. 4). La administración de la primaquina debe hacerse siempre con base en el peso corporal del pacientes, ya que el margen entre las dós i s terapéuticas y tóxicas es muy pequeño y la susceptibilidad individual es variada. Los efectos colaterales más importantes están representados por la metahemoglobinemia, caracterizada clínicamente por cianosis de labios y piel y la anemia hemolitica aguda en pacientes con deficiencia de la enzima glucosa-6-fosfato deshidrogenasa. Esta enzima es requerida para reducir el NADP (Nicotinamina Acienina Dinocleotido Fosfato) a NADPH en el eritrocito, sin el cual 
CUADRO 3.

\section{PLĀSMODIUM FALLCIPARUM \\ TRATAMIENTO DE LA INFECCION AGUDA COMPLICADĀ}

\begin{tabular}{|c|c|c|c|c|}
\hline \multirow{2}{*}{$\begin{array}{l}\text { CARACTERISTICAS } \\
\text { DE LA INFECCION }\end{array}$} & \multirow{2}{*}{ DROGAS } & \multirow{2}{*}{$\begin{array}{l}\text { VIA DE } \\
\text { ADMON. }\end{array}$} & \multicolumn{2}{|c|}{ DOSIS } \\
\hline & & & ADULTOS & NIN̄OS \\
\hline $\begin{array}{l}\text { 1. Número de parósitos supe- } \\
\text { rior a } 100.000 / 41 \text { sangre. } \\
\text { - Vómito y/o diarrea seve } \\
\text { ros. } \\
\text { - Compromiso cerebral. }\end{array}$ & $\begin{array}{l}\text { Diclorohidrato de Qui } \\
\text { nina. } \\
\text { Sulfadoxina (Fanasil) } \\
\text { Pirimetamina * }\end{array}$ & $\begin{array}{l}\text { Oral } \\
\text { Oral }\end{array}$ & $\begin{array}{l}7-10 \mathrm{mg} / \mathrm{kg} / 500 \mathrm{ccDex} \\
\text { trosa cada } 8 \text { horas. } \\
\text { Dosis única: } 1.500 \mathrm{mg} . \\
\text { Dosis única: } 75 \mathrm{mg}\end{array}$ & $\begin{array}{l}7-10 \mathrm{mg} / \mathrm{kg} / \mathrm{cada} 8 \\
\text { horas. } \\
\text { Dosis única: } 25 \mathrm{mg} / \mathrm{kg} \\
\text { Dosis única: } 1 \mathrm{mg} / \mathrm{kg}\end{array}$ \\
\hline $\begin{array}{l}\text { 2.- Compromiso hepático se } \\
\text { vero. } \\
\text { - Insuficiencia renal oguda } \\
\text { (IRA). }\end{array}$ & $\begin{array}{l}\text { Diclorohidrato de Quj } \\
\text { nina. } \\
\text { Sulfadoxina (Fanasil) * } \\
\text { Pirimetamina. * }\end{array}$ & $\begin{array}{l}\text { I.V. } \\
\text { Oral } \\
\text { Oral }\end{array}$ & $\begin{array}{l}10 \mathrm{mg} / \mathrm{kg} / 24 \text { horas. } \\
\text { Dosis ánica: } 1.500 \mathrm{mg} \\
\text { Dosis única: } 75 \mathrm{mg}\end{array}$ & $\begin{array}{l}\text { I/3 dosis normal } / 24 \mathrm{hs} \\
\text { Dosis única: } 25 \mathrm{mg} / \mathrm{kg} \\
\text { Dosis única: } 1 \mathrm{mg} / \mathrm{kg}\end{array}$ \\
\hline
\end{tabular}

CUADRO 4.

INFECCIONES POR PLASMODIUM VIVAX

\begin{tabular}{|c|c|c|c|}
\hline \multirow{2}{*}{ DROGAS } & \multirow{2}{*}{$\begin{array}{l}\text { VIA DE } \\
\text { ADMON. }\end{array}$} & \multicolumn{2}{|c|}{ DOSIS } \\
\hline & & ADULTOS & NIN̄OS \\
\hline \multirow[t]{5}{*}{ Cloroquina (base) } & Oral & Dosis total: $1.500 \mathrm{mg}$ & Dosis inicial: $10 \mathrm{mg} / \mathrm{kg}$ \\
\hline & & Dosis inicial: $600 \mathrm{mg}$. & 6 horas: $5 \mathrm{mg} / \mathrm{kg}$ \\
\hline & & 6 horas: $300 \mathrm{mg}$. & 24 horas: $5 \mathrm{mg} / \mathrm{kg}$ \\
\hline & & 24 horas: $300 \mathrm{mg}$. & 48 horos: $5 \mathrm{mg} / \mathrm{kg}$ \\
\hline & & 48 horas: $300 \mathrm{mg}$ & \\
\hline Primaquina (base) & Oral & Dosis: $15 \mathrm{mg} /$ dia $/ 14$ dias. & Dosis: $0,3 \mathrm{mg} / \mathrm{kg} / \mathrm{dia} / 14$ dias \\
\hline \multicolumn{4}{|l|}{ Recrudescencia: } \\
\hline Cloroquina (base) & Oral & Dosis: $600 \mathrm{mg} / \mathrm{sem} / 8 \mathrm{sem}$. & Dosis: $10 \mathrm{mg} / \mathrm{kg} / \mathrm{sem} / 8 \mathrm{sem}$. \\
\hline Primaquina (base) & Oral & Dosis: $45 \mathrm{mg} / \mathrm{sem} / 8 \mathrm{sem}$. & Dosis : $0,9 \mathrm{mg} / \mathrm{kg} / \mathrm{sem} / 8 \mathrm{sem}$. \\
\hline
\end{tabular}


la célula es incapaz de mantener sus concentraciones normales de glutation reducido (GSH). Aunque sus funciones no son muy claras, el GSH parece importante en la protección del glóbulo rojo contra una variedad de agentes químicos y físicos (10).

Algunos casos tratados en el esquema propuesto presentan recaídas, sin haberse determinado si existe o no resistencia del $\mathbf{P}$. vivax a las drogas. Si la infección se presenta nuevamente después de 6 a 8 semanas de concluido el tratamiento completo con la primaquina, probablemente la población de parásitos tenía una baja sensibilidad a las drogas anłimaláricas. En estos pacientes es recomendable administrar un segundo ciclo del esquema inicial o prolongar el tratamiento por un periodo de 8 semanas, a las dósis semanales sugeridas tanto de la cloroquina como de primaquina.

\section{R E S U M E N}

El tratamiento de las infecciones producidas por el P. falciparum resistente a la cloroquina presenta diferentes alternativas de acuerdo con el cuadro clínico del paciente, el grado de resistencia de los parásitos a los antimalaricos y el nivel de inmunidad desarrollado a través de previas infecciones. La determinación del número de parásitos/m.c.l.sangre en la muestra inicial del paciente, es uno de los parámetros básicos en la selección del tratamiento y su vía de administración. Cuando el paciente tolere la vía oral y tenga recuentos inferiores a 100.000 parásitos por m.c.t. de sangre, la medicación con el sulfato de quinina en ciclos cortos de 3 días y asociada a la combinación de sulfa-pirimetamina, es el tratamiento de elección.

Infecciones con recuentos superiores y/o complicaciones de la infección, deberán ser tratadas por via parenteral a la dós is de 7-10 $\mathrm{mg}$. de diclorohidrato de quinina cada 8 horas, con la adición posterior de la sulfa-pirimetamina. Diferentes combinaciones de drogas son sugeridas en caso de una recrudescendencia de la infección a los tratamientos descritos anteriormente, evaluando en forma critica la resistencia múltiple del P. falciparum a las drogas antimaláricas.

\section{S U M M A R Y}

The treatment of $\mathbf{P}$. falciparum resistant to chloroquine will depend upon the clinical features of acute falciparum infection, the susceptibility of the parasites to antimalarial drugs and the level of immunity of the host. Serial parasite counts/m.c.l of blood should be made in order to select the treatment and evaluate the efect on the parasitoemia.

Patients with parasite counts below 100.000 im.t. of blood should be treated with quinine sulfate for three days in association with sulfadoxine-pyrimethamine. If the patient has a high parasite count ( $100.000 /$ m.c.l of blood) or a complicated infection, quinine dihydrochloride should be administered as a slow intravenous infusion, at a dose of 7-10 $\mathrm{mg} / \mathrm{kg}$ every 8 hours. A variety of treatment regimens combinign different antimalarials are recommended, if there is a subsequent recurdescence of the infection to precious courses. Special care is needed when treating acute infections with multidrug-resistant parasites, in orden to evaluate the reason why an infection fails to respond to standard antimalarial therapy.

\section{AGRADECIMIENTOS}

El autor desea expresar sus agradecimientos al Dr. K. H. Rieckmann, Director de la División de Medicina Tropical, Universidad de New México, U.S.A., por su valiosa colaboración en la evaluación de este documento.

\section{R E F E R E N C I A S}

1. Moore, D.V.y Lanier, S.E. Observations on two $P$. falciparum infections with an abnormal response to chloroquine. Am. J. Trop. Med. Hyg. 1961. 10 (1), 5-9.

2. Young, M. y. Moore, D.V. Chloroquine resistance in $P$. falciparum Am. J. Trop. Med. Hyg. 1961. 10. 317-320.

3. Young, M. Amodiaquine and Hydroxicloroquine resistance in $P$. falciparum Am. J. Trop. Med. Hyg. 1961. 10 (5). 689-693. 
4. López - Antuñano, F.J. Medición de la susceptibilidad del P. falciparum a la cloroquina por un método simple in-vitro en Brasil, Colombia, Haití y México. Congreso de la Federación Latinoamericana de Parasitología y Medicina Tropical. 1973. Medellín, Colombia, S.A.

5. WHO Scientific Group. Chemotherapy of Malaria and Resistance to Antimalarials. Wld. Hlth. Org. Technical Report Series. $529: 30-31$.

6. Don Michael, T.A. y Aiwazzadeh, S. The effects of acute chloroquine paisoning with special reference to the heart. American Heart Journal. $79: 831-842$.
7. Schmidt, L. H. y col. Potential utility of other 4-Aminoquinolines in treating Plasmodium falciparum infections resistant to chloroquine. American Society of Tropical Medicine and Hygiene. 1970. San Francisco.

8. Rieckmann, K.H. Determination of the drug sensitivity of $P$. falciparum. J. Amer. Med. Assoc. 1971. 217 (5) : 573-578.

9. Weniger, H. Review of side effects and toxicity of pirimethamine. WHO/MAL. 1979 $79 / 907$.

10. Weniger, H. Toxicity and side effects of primaquine and other 8-Aminoquinolines. WHO/MAL. 1979. 79/905. 